\title{
Fuzzy Logic Based Energy Aware Routing Protocol for Internet of Things
}

\author{
S.Sankar \\ Research Scholar, School of Computer Science and Engineering, VIT University, Vellore, Tamilnadu, India \\ E-mail: sankar.s@vit.ac.in \\ P.Srinivasan \\ Associate Professor, School of Information Technology and Engineering, VIT University, Vellore, Tamilnadu, India \\ E-mail: srinivasan.suriya@vit.ac.in
}

Received: 11 October 2017; Accepted: 09 February 2018; Published: 08 October 2018

\begin{abstract}
Maximizing the network lifetime is one of the major challenges in Low Power and Lossy Networks (LLN). Routing plays a major role in LLN, for minimizing the energy consumption across the network nodes. IPv6 Routing Protocol for Low Power and Lossy Networks (RPL) is a standardized routing protocol for LLN. Though, RPL fulfilled the necessity of LLN, several issues like increasing the energy efficiency, quality of service and the network lifetime are to be focused. In LNN, the inefficient route selection results in increased network traffic, energy depletion and packet loss ratio across the network. In this paper, we propose a fuzzy logic based energy aware routing protocol (FLEARPL), which considers the routing metrics load, residual energy (RER) and expected transmission count (ETX) for the best route selection. FLEA-RPL applies fuzzy logic over these metrics, to select the best route to transfer the network data efficiently. The COOJA simulator is used to assess the efficiency of the proposed FLEA-RPL. The FLEA-RPL protocol is compared with similar protocol standard RPL, MRHOF (ETX) based RPL (MRHOFRPL) and FL-RPL. The simulation result shows that FLEA-RPL improves the network lifetime by $10-12 \%$ and packet delivery ratio by $2-5 \%$.
\end{abstract}

Index Terms - Internet of Things, IPv6 Routing Protocol for Low-Power and Lossy Networks, Fuzzy Logic, Load, Energy Efficiency.

\section{INTRODUCTION}

Internet of Things (IoT) is a recent and emerging research area of social, technical and economic significance [1]. It enables the machine to machine communication over the internet, without human involvement [2-4]. Nowadays, the people are using IoT application such as smart home, smart city, healthcare, wearable, smart grid, connected car, smart retail, smart supply chain and smart farming [3].

LLN is a network, which composed of resource constrained devices interconnected by the wireless links[5]. Routing plays a vital role for energy conservation in Low power and lossy networks (LLN). In LLN, the routing protocol has the constraints like low data processing capacity, limited memory and energy. Moreover, LLN routing design need to self organize the network nodes; by healing itself without manual involvement [6]. Thus, the conventional routing protocol is not suitable for LLN. IETF standardized IPv6 routing protocol for low power and lossy networks (RPL) for LLN [7].

In LLN, if the participant node needs to join the DODAG, it should consider different routing metrics for the best route selection to transfer the data. In this paper, we propose fuzzy logic based energy aware routing protocol (FLEA-RPL), which considers the routing metrics Load, RER and ETX, to select the best route, to transfer the data efficiently. Thus, it decreases the multipoint to point data traffic in upward routing, point to multipoint data traffic in downward routing.

The paper is organized as follows: Section 2 describes the related work. Section 3 describes the Proposed Routing protocol design. Section 4 represents the performance evaluation. Finally, we conclude and discuss the future work in section 5 .

\section{RELATED WORKS}

In this section, we present the description of RPL routing protocol and recent research carried on energy aware RPL.

\section{A. Overview of $R P L$}

RPL is a proactive routing protocol and it follows the Destination oriented Acyclic Graph (DODAG). In DODAG, the participant node sends the data to DODAG root, through parent node and the root node finds the route, to forward the data to the destination node. Upward root indicates the edge direction towards the root and downward root indicates the edge direction away from the root. RPL protocol generates more than one RPL instances in a network and each instance contains more than one DODAG. RPL rank indicates that how long distances from DODAG root to parent. The DODAG 
node has storing and non storing mode. By default, the root node has storing mode and it maintains the routing table about the entire network. In DODAG, the root node gives the right, to some node and it can maintain the part of network routing information. In storing mode, the node can collect and forward the data to other node. In non storing mode, the node can simply forward the data to other node.

RPL has four control messages namely, DODAG Information Solicitation (DIS), Information Object (DIO), Advertisement Object (DAO) and Advertisement ObjectAcknowledgement (DAO-ACK). Initially, the DODAG request process is carried out in two ways. i. participant node sends the DIS request to DODAG. ii. DODAG sends the DIO request messages to all participant nodes. The DODAG enables the trickle timer; the participant node needs to send the DAO control messages to DODAG within the time interval. Then, the DODAG sends the DAO-ACK control messages to all participant nodes [8].

\section{B. Energy Aware RPL}

Recently, many research works has done on energy aware routing in RPL, which minimizes the node energy consumption, to improve the network lifetime. Most of the Energy aware RPL work is focused on single routing metric, composite metric using average weight values and composite metric using fuzzy logic.

Oana Lova et'al [9] proposed the expected lifetime as routing metric to improve the network lifetime. The main objective of this work is to find the bottleneck of node and spread the network traffic among the network nodes. It achieves the reliability and stability of the network. Mamoun Qasem et'al [10] introduced a new objective function, to balance the traffic load over the network nodes. It is mainly focused on energy efficiency and it avoids the data transmission failures over the network. Zhenfei Wang et'al [11] designed an improved RPL routing protocol for WSN. In IRPL, they proposed an objective function namely, Life Cycle Index (LCI) for path selection. The LCI is considered the node metrics namely, hop count and residual energy and the link metric namely, throughput, packet loss ratio and link quality. IRPL redesigned the parent selection strategy for rank calculation and it is adopted into DODAG structure. Thus, IRPL is improved the overall network lifetime.

Ali Hassan et'al [12] proposed a composite metric based RPL, which consider the routing metrics residual energy and battery discharge index, to improve the network lifetime. It avoids the battery depletion near to the sink node, for transfer the data from source to destination nodes. Mohammad Nassiri et'al [13] proposed an efficient and dynamic parent selection in RPL routing, which considers the composite metrics remaining energy and load, to select the multiple parent and transfer the data efficiently. In RPL, It is modified the cluster tree in MAC layer, so it is extended the lifetime of the network. Hongliang Tian et'al[14] introduced the quality of information aware RPL (QoI-RPL) and it is improved the standardized RPL in two ways, such as neighbor list and information of DIO and QoI of data are used to detect the event, for optimizing the topology and reduce energy consumption in RPL.

In [4] proposed energy aware routing protocol, which considers load and battery discharge index, to select the best path for transfer the data effectively. It avoids the early battery depletion near to sink node and also suitable for small scale networks. Nikesh man Shakya et'al [15] introduced a smart energy efficient objective function to develop the reliability and energy efficiency for smart metering application. It is considered the routing metric such as ETX and estimated remaining lifetime for improving the network lifetime. In [7] evaluated the performance of IRPL for wireless sensor networks. It introduced the composite routing metrics remaining energy and link quality, to select the best route, for transfer the data from source to destination.

Olfa Gaddour et'al [16] proposed QoS aware RPL routing for LLN. It considered the routing metrics end-toend delay, expected transmission count, hop count and link quality level. It is applied the fuzzy logic over these metrics to select the best path for transfer the data efficiently over the network nodes. Sheeraz A.Alvi et'al [17] proposed a new objective function, which contain the routing metrics hop count and ETX, for improving the network lifetime.

\section{Proposed Routing Protocol DESIGN}

In this paper, we propose FLEA-RPL and it is an improved version of standard RPL. FLEA-RPL applies the fuzzy logic over the routing metrics Load, RER and ETX to select the best route, to transfer the data efficiently. The above mentioned routing metric evaluates the quality of preferred parent and it compares with set of preferred parent. Finally, it chooses the best parent among the preferred parent in DODAG. The participant node sends the data to DODAG root through best parent.

\section{A. Metrics of Interest}

\section{A1. Residual Energy}

Residual energy (RER) measures the amount of available energy in the network node. Residual energy indicates that the variation of initial energy and current energy of node RER(Ni) [12]. The residual energy calculation formula is given in (1).

$$
R E R(N i)=\frac{\text { Current_Energy }}{\text { Initial_Energy }}
$$

\section{A2. Load}

Network traffic or traffic load is an amount of data moving across the network at specific instant of time. Mamoun Qasem et'al has introduced a load balancingobjective function for LLN and it is standardized by IETF. Load balance is a technique and it balances the data traffic based on number of children present in the 
candidate parent node [17]. During the parent selection, it takes into account the number of children count in the parent. The traffic load calculates from (2) and (3).

The path load calculates from cumulative count of children node in the entire route.

$$
\operatorname{Load}(P x)=\sum_{i=1}^{n} N o d e_{-} \text {Traffic_Load }(i)
$$

Node traffic calculates from child count of the respective node.

$$
\text { Node_Traffic_Load }(i)=\sum_{j=1}^{n} \operatorname{child} \_ \text {count }(j)
$$

\section{A3. Expected Transmission Count}

Expected transmission count is a wireless link metric and it predicts the link quality from number of transmission and including retransmission of the data delivery [18]. The ETX metric calculates from (4) and (5).

\section{- Link ETX}

Link ETX represents the forward and reverse data delivery of the particular link. The forward data delivery (Df) represents the probability of data packet that successfully reached at recipient and reverse data delivery (Dr) represents the probability that acknowledgement packet received successfully at the sender. The link ETX calculates from (4).

$$
\operatorname{ETX}(N i)=1 /(D f * D r)
$$

\section{- $\quad$ Route ETX}

Route ETX finds the link quality of particular path PX. The Route ETX calculates from (5).

$$
\operatorname{ETX}(P x)=\sum_{i=1}^{n} \operatorname{ETX}(N i)
$$

\section{B. Fuzzy logic based composite routing metric in RPL}

FLEA-RPL applies the fuzzy logic over the routing metrics Load, RER and ETX, to select the best route, to transfer the data efficiently. Fuzzy logic is a multi valued logic and its value between 0 and 1 . During the parent selection, fuzzification and defuzzification process is carried out, for selecting the quality of parent node, to transfer the data from source to destination.

\section{B1. Fuzzification}

Fuzzification is a process of convert the crisp input value into fuzzy set. In FLEA-RPL, the routing metrics are Load, RER and ETX, to select the best path, to transfer the data. In fuzzy logic, the important terminologies are linguistic variable and membership function.

\section{- $\quad$ Linguistic Variable}

Linguistic variable is a structure of language and it is divided into a number of sub fields. It belongs to set and the values between true and false. The linguistic variable holds the input and output of routing metrics. It represents the value in the form of words rather than numeric.

Linguistic variable of load contains three sets according to the position of DODAG, light, normal and heavy. Fig. 1 shows that the value of load is less than or equal to 3, a fuzzy subset of node is "Light" of the DODAG and membership value is 1 . The value of load between 3 and 6 , the membership value is decreasing gradually from 1 to 0 . Likewise, we consider the linguistic variable "Normal" and "Heavy" as per the diagram.

Linguistic variable of RER contains three sets according to the position of DODAG, low, average and full. Fig. 2 shows that the value of the RER is less than or equal to 75, a fuzzy subset of node is "Low" of the DODAG and membership value is 1 . The node value between 25 and 75, the membership value is from 1 to 0 . Likewise, we follow the linguistic variable "Average", "Full" as per the diagram.

Linguistic variable of ETX contains three sets according to the position of DODAG, short, average and long. Fig.3 shows that the value of ETX is less than or equal to 10, a fuzzy subset of node is "Short" of the DODAG and membership value is 1 . The value of ETX between 10 and 30, membership value is decreasing gradually from 1 to 0 . Likewise, we follow the linguistic variable "Average", "Long" as per the diagram.

Linguistic variable for quality of neighbor node contains seven sets, awful, low bad, bad, low good, good, very good and excellent. The quality of the values between 0 and 100 assigns to preferred parent node. Fig.4 shows that the quality of neighbor is less than or equal to 12 , a fuzzy subset of node is "Awful" of the DODAG and membership value ranges from 0 to 1 . Likewise, it finds the membership values from different quality of preferred node value.

\section{- Membership Function}

A membership function evaluates the linguistic variable, to provide the accurate measurement of input and output variable. Fig.1-4 shows that the membership functions of load, RER and ETX. We have chosen the trapezoidal shape membership function for input variable and triangle shape membership function for output variable in the fuzzy logic system [19-21]. The membership function and threshold values can be finetuned based on the application requirements.

Load membership function indicates the data traffic across the network nodes. Liang Zhao et'al introduced Neighborhood Load Routing, to detect the network traffic load. It is represented the network load as light, normal and heavy [22]. The linguistic variable Light (Load) membership function can be written from (6). 


$$
\text { Light }(\text { Load })=\left\{\begin{array}{l}
1 \text { if load } \leq 3 \\
\frac{\text { Load }-3}{6-3} \quad \text { if } 3<\text { load }<6 \\
0 \quad \text { if load } \geq 6
\end{array}\right.
$$

Likewise, we can write remaining linguistic variables such as normal and heavy. In addition, we can represent the membership function of RER and ETX. Fig.1 shows that membership function of Load metric and it ranges from 0 to 20 .

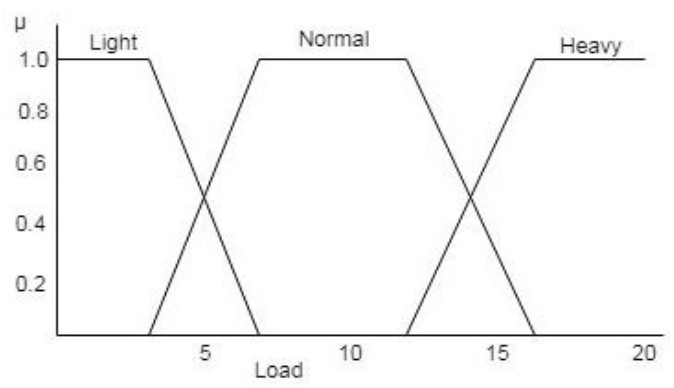

Fig.1. Input variable of the Load

Residual energy membership function measures the remaining energy from the battery. T. Winter et'al proposed a RFC 6550 for LLN and it is standardized by IETF. It specifies the energy ranges from 0 to 255 [8]. Fig. 2 shows that residual energy membership and its values from 0 to 1 . During parent selection process, the participant node selects the high remaining energy from preferred parent node.

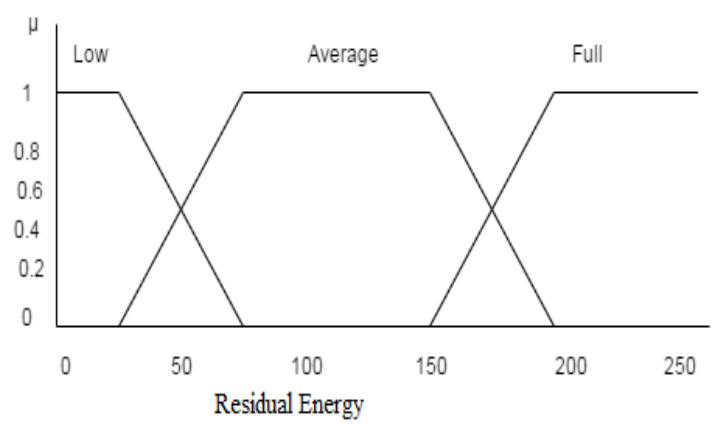

Fig.2. Input variable of the RER

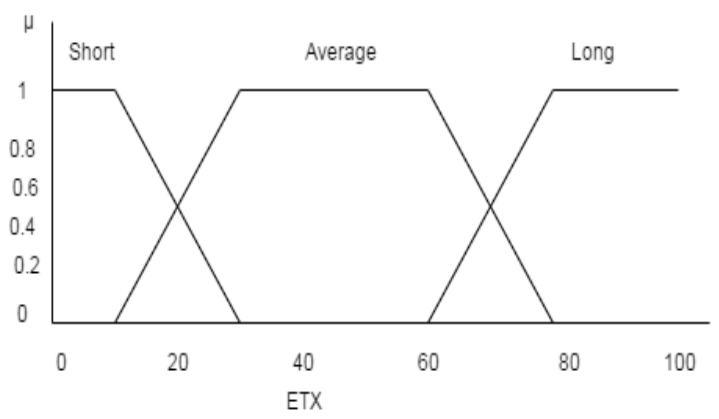

Fig.3. Input variable of the ETX

ETX indicates the quality of the node link and it is one of the prominent routing metric in LLN. Fig. 3 shows that
ETX metric ranges from 0 to 100 . We have set the ETX ranges and membership values from Olfa Gaddour et'al introduced QoS aware fuzzy logic based RPL [16].

Quality of the neighbor node membership function ranges from 0 to 1 . The quality ranges between 0 and 100 that assigns to the neighbor node. The output variable and its value ranges are decided from olfa Gaddour et'al introduced QoS aware fuzzy logic based objective function for RPL [6]. Fig.4 shows that quality neighbor node.

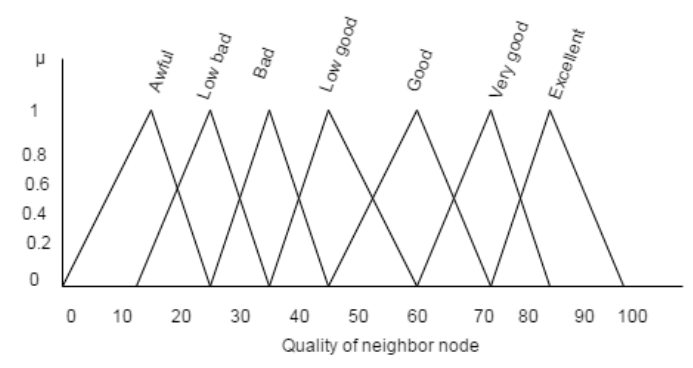

Fig.4. Output variable of neighbor quality

Table 1. Fuzzy Rule Base

\begin{tabular}{|c|c|c|c|c|}
\hline S.No & Load & $\begin{array}{c}\text { Residual } \\
\text { Energy }\end{array}$ & ETX & Quality \\
\hline 1 & Light & Full & Short & Excellent \\
\hline 2 & Light & Full & Average & Very good \\
\hline 3 & Light & Full & Long & Good \\
\hline 4 & Light & Average & Short & Very good \\
\hline 5 & Light & Average & Average & Good \\
\hline 6 & Light & Average & Long & Good \\
\hline 7 & Light & Low & Short & Good \\
\hline 8 & Light & Low & Average & Bad \\
\hline 9 & Light & Low & Long & Low bad \\
\hline 10 & Normal & Full & Short & Very good \\
\hline 11 & Normal & Full & Average & Good \\
\hline 12 & Normal & Full & Long & Bad \\
\hline 13 & Normal & Average & Short & Good \\
\hline 14 & Normal & Average & Average & Low good \\
\hline 15 & Normal & Average & Long & Low bad \\
\hline 16 & Normal & Low & Short & Bad \\
\hline 17 & Normal & Low & Average & Low bad \\
\hline 18 & Normal & Low & Long & Bad \\
\hline 19 & Heavy & Full & Short & Good \\
\hline 20 & Heavy & Full & Average & Bad \\
\hline 21 & Heavy & Full & Long & Good \\
\hline 22 & Heavy & Average & Short & Bad \\
\hline 23 & Heavy & Average & Average & Low Bad \\
\hline 24 & Heavy & Average & Long & Bad \\
\hline 25 & Heavy & Low & Short & Low bad \\
\hline 26 & Heavy & Low & Average & Bad \\
\hline 27 & Heavy & Low & Long & Awful \\
\hline
\end{tabular}

\section{B2. Fuzzy Rules}

In FLEA-RPL, the input fuzzy set is a combination of different metrics Load, RER and ETX. Each metric is an individual fuzzy variable. Rule base consists of $3^{3}=27$ fuzzy rules, which is based on the fuzzy input variable and fuzzy membership function. According to application 
requirement, we can fine-tune the fuzzy rule. Table. 1 represents the fuzzy rule base, in that first column represent the rule count, column 2 to 4 represents input fuzzy variable and end of the column represents output fuzzy variable in term of "Quality".

The output fuzzy set "Quality" indicates the neighbor or preferred parent quality. It contains seven elements in fuzzy set from "awful" to "excellent". The quality of neighbor value ranges between 0 and 100 and it finds the neighbour quality of each participant node. Fuzzy rule evaluates the fuzzy input using mamdani model [23]. It follows the minimum operator as composition function and maximum operator as aggregation function.

\section{B3. Defuzzification}

Defuzzification is a process of convert the fuzzy input into crisp set. It provides single crisp value from several membership functions and its value ranges between 0 and 100. We have chosen the weighted average method for defuzzification [24]. Equation (7) represents the weighted average method in mathematically, the crisp set $\mathrm{R}$ from the fuzzy region $B$.

$$
\mathrm{R}=\frac{\sum_{i=1}^{N} W_{i} \times \mu_{B}\left(W_{i}\right)}{\sum_{i=1}^{N} \mu_{B}\left(W_{i}\right)}
$$

Where $\mathrm{N}$ is number of fuzzy rules occurred in the fuzzy inference system, $\mathrm{W}_{\mathrm{i}}$ is domain value respective rule " $i$ " and $\mu_{B}$ indicates the predicate truth in domain.

For an instance, the neighbor node contains the load value is 2, RER value is 175 and ETX value is 10. As per the FLEA-RPL membership function, the Load metric fuzzy set or linguistic variable value is "light" and membership function value is 1. Likewise, the RER fuzzy set values are "Average and full" and Membership values are 0.5 and 0.5 respectively. Moreover, ETX metric fuzzy set value is "Short" and membership value is 1 . Hence, two fuzzy rules i.e. rule 1 and 4 matched and it activates the results "Excellent" and "Very good". By applying fuzzification process, the result of rule 1 is 0.5 and rule 4 is 0.5 . The defuzzified crisp value $(R)$ is calculated in below.

$$
R=\frac{(0.5 \times 72+0.5 \times 84)}{(0.5+0.5)}=78
$$

The participant node selects the best parent from preferred parent node quality.

\section{Rank Calculation}

The rank of node "N" calculates from rank of parent node of " $N$ " and rank increase. The rank increase calculates from step and MinHopRankIncrease. MinHopRankIncrease value is standardized and default value is 256 . The step value calculates from routing metrics load, ETX and RER using fuzzy logic. The rank calculates from (9) and (10).

$$
\operatorname{Rank}(\mathrm{N})=\text { Parent_Rank(N)+Rank_increase }
$$

Rank_increase $=$ Step $\times$ Min_Hop_Rank_Increase

\section{Parent Selection Process}

The DODAG request process is carried out in two ways. i). participant node sends the DIS request to DODAG. ii). Periodically, DODAG will send the DIO control message to entire participant node. The proposed FLEA-RPL routing protocol considers the routing metrics load, ETX and RER, which applies the fuzzy logic over these routing metric, to select the best parent among the preferred parent across the network node.

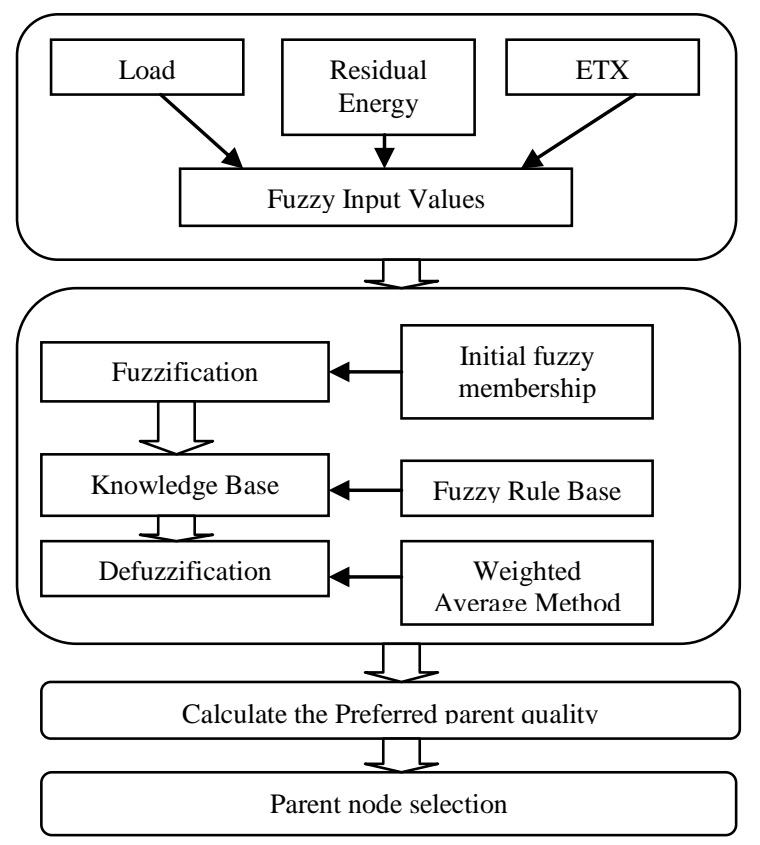

Fig.5. Parent selection mechanism

Before sending the DIO control message, the DODAG activates timer; the counter $\mathrm{C}$ value is initialized as zero. The trickle time interval size is denoted as "I" and it ranges from $I_{\min }$ to $I_{\max }$. The $I_{\min }$ value is $12 \mathrm{~ms}$ and $I_{\text {doubling }}$ value is $10 \mathrm{~ms}$. The DODAG sends the DIO control messages to the entire participant node. Finally, DODAG accepts the participant's confirmation and it sends the DAO-ACK messages to the respective participant node. If the participant node is getting delay to send the DAO messages to DODAG, it reset the trickle timer and again does the same process. The parent selection process and algorithm is given in below.

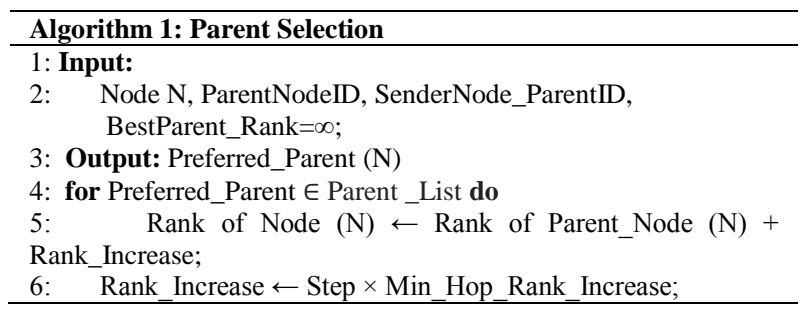




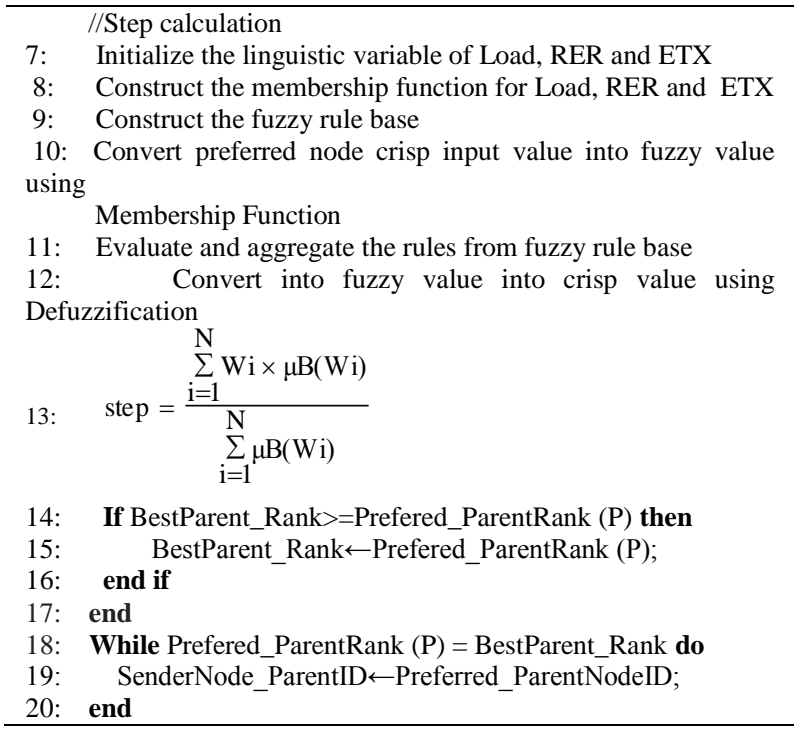

\section{PERformance EVAluation}

\section{A. Simulation Setting and Parameter}

The purpose of the simulation is to reveal, how FLEARPL protocol provides the better performance, it is compared with other similar routing protocol RPL, MRHOF-RPL and FL-RPL in LLN. The proposed FLEA-RPL protocol conducted the simulation using COOJA simulator. We have conducted the simulation several times. It consists of $100 \mathrm{RPL}$ routers with 1 DODAG root over the square environment $(600 \mathrm{~m} \times$ $600 \mathrm{~m})$. The experimental nodes located randomly and connected in the LLN. Our simulation is considered the TmoteSky and it is provided the radio channel is 26 and the maximum transmission power level is $0 \mathrm{dBm}$. In each time of simulation, every 60 seconds RPL router sends 1 data packet into DODAG root. However, LLN changes the topology of every 60 seconds. Table. 2 shows that the simulation configuration for this experiments.

Table 2. Simulation Configuration

\begin{tabular}{|l|l|}
\hline Parameter & Value \\
\hline Operating System & Contiki 2.7 \\
Node Type & Tmote sky \\
DIO interval doubling & 10 \\
Minimum DIO interval & 12 \\
Routing Protocol & RPL \\
Radio Environment & Unit Disk Graph Medium \\
MAC/Adaptation Layer & ContikiMAC/6LowPAN \\
Simulation Duration & 2 Days \\
Number of Nodes & 100 RPL Router + \\
& 1 DODAG root \\
Full Battery & $3000 \mathrm{~mJ}$ \\
Data Packet Timer & $60 \mathrm{sec}$ \\
Transmission Range & $600 \times 600 \mathrm{~m}^{2}$ \\
RPL Parameter & MinHopRankIncrease $=256$ \\
\hline
\end{tabular}

\section{B. Performance Metrics}

The evaluation of FLEA-RPL based on the following performance metrics.

\section{- Average Number of Parent Changes}

It denotes, how many times the node is changed of its parent in a DODAG. It measures the network stability. The minimal number of parent changes leads to stable topology.

\section{- Average End-to-End Delay}

It indicates the time delay between the data packet forward from sender (DODAG node) and received by receiver (DODAG root).

\section{- Average Packet Loss Ratio}

It indicates the ratio of total amount of failure data packet at destination and total number of data packets sent by the sender.

\section{- $\quad$ Average Remaining Energy}

It estimates the network lifetime, which indicates the average residual or remaining energy present in the network nodes.

\section{Evaluation Results}

In this section, we evaluated the performance of FLEA-RPL and it is compared with other similar routing protocol RPL, MRHOF-RPL and FL-RPL.

\section{C1. Number of Parent Changes}

We measured the parent changes in FLEA-RPL and it is compared with RPL, MRHOF-RPL and FL-RPL. Fig.6 shows that the routing protocol RPL, MRHOF-RPL and FL-RPL and FLEA-RPL of the average numbers of parent changes rate per hour are 0.2, 0.25, 0.27 and 0.26 respectively. The higher number of parent change value indicates the topology instability. In FLEA-RPL, the number of parent change value is slightly high but it is smaller than FL-RPL.

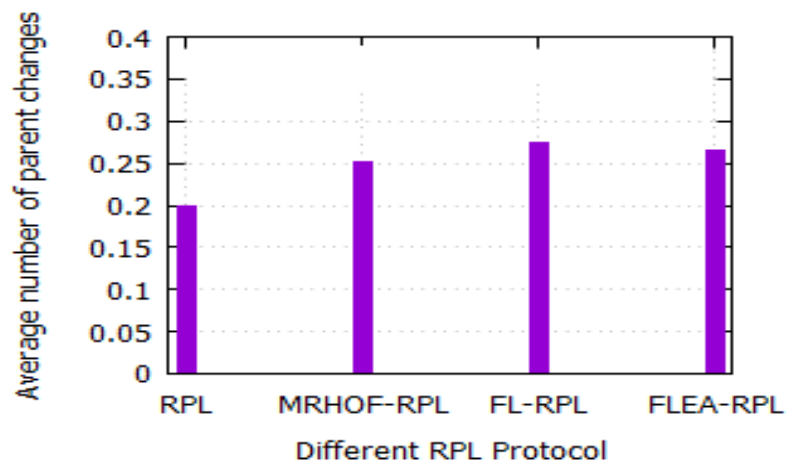

Fig.6. Average number of parent changes with different RPL protocol

\section{C2. Average End-to-End Delay}

Fig.7 shows that an average end-to-end delay (latency) and it is compared with RPL, MRHOF-RPL and FL-RPL and FLEA-RPL. Average end-to-end delay indicates the time duration between the packet transmissions from source to destination. RPL has provided more latency than MRHOF-RPL and FL-RPL. Standard RPL has provided the shortest path, high latency and congestion happened in RPL. However, FLEA-RPL has provided the low average end-to-end delay (latency) than similar other routing protocol RPL, MRHOF-RPL and FL-RPL. In FLEA-RPL, the maximum delay deals 2.9 seconds for 10 
hops versus 3.8 seconds for RPL. This parameter proves that FLEA-RPL reduced the number of hop count and minimized the average end-to-end delay compared with other similar RPL protocol.

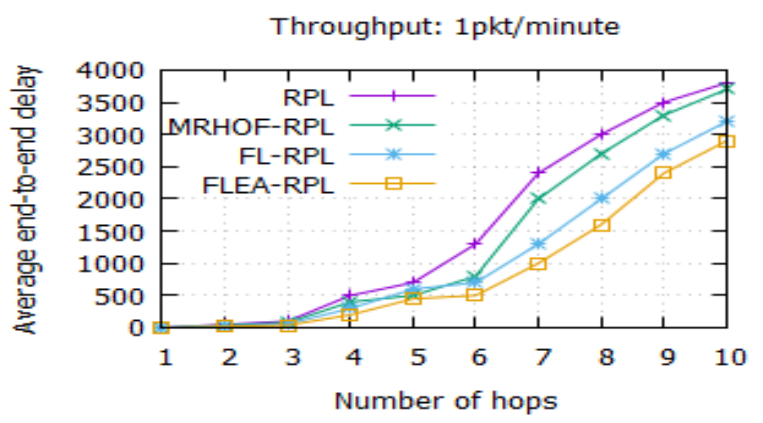

Fig.7. Average end-to-end delay (latency) with different objective function

\section{C3. Network Lifetime}

In this simulation, we monitored the remaining energy of entire node in a network and FLEA-RPL is compared with other similar RPL. We conducted the simulation for two days. We adjusted the throughput range from 1 packet / minute to 6 packet / minute. Fig. 8 shows that an average remaining energy distribution of nodes in the network with 1 packet per minute.

For instance, the simulation conducted for two days with RPL and throughput is 1 packet/ minute. The network contains that $23 \%$ of the node remaining energy is under $80 \%, 31 \%$ of node remaining energy is between $81 \%$ and $85 \%, 26 \%$ of the node remaining energy is between $86 \%$ and $90 \%, 10 \%$ of node remaining energy is between $91 \%$ and $92 \%$. The remaining energy decides the network survivability. In RPL, the nodes are maintaining different remaining energy. Therefore, most of node will be happened the early battery exhaustion.

In FL-RPL, the network contains $86 \%$ of node remaining energy is between $84-87 \%$ and $14 \%$ node remaining energy is $90 \%$ of the battery.

FLEA-RPL optimized the parent selection and improved the network life than objective function (OF-FL) based FL-RPL. The network node contains $90 \%$ of the node remaining energy is between 84 to $87 \%$ of their battery and $10 \%$ of the node remaining energy is $92 \%$. The FLEA-RPL improves the network lifetime across the network nodes.

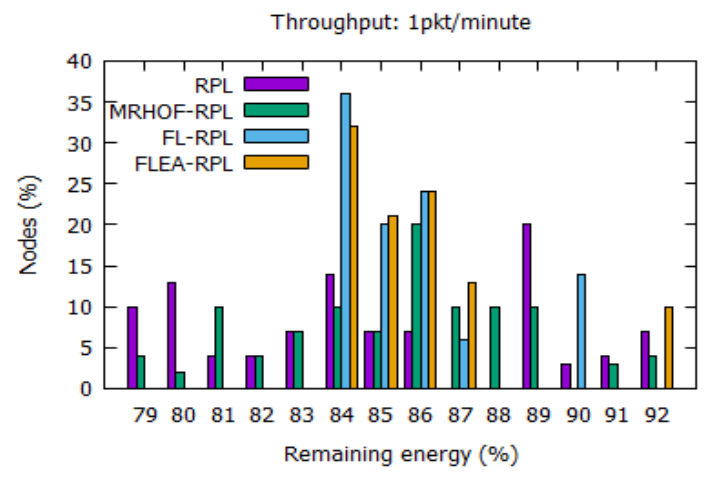

Fig.8. Remaining power distribution for 1packet/minute
Fig.9 shows that the average remaining energy distribution of nodes in the network with 6 packet per minute. For instance, the network with objective function (OF-FL) based FL-RPL contains $86 \%$ of the node remaining energy is between $62-67 \%$ and $14 \%$ of the node remaining energy is $70 \%$ of the node battery.

In FLEA-RPL, the network contains $90 \%$ of the node remaining energy is between $62-66 \%$ of their battery and $10 \%$ of the node remaining energy is $72 \%$ of the node battery. The FLEA-RPL is demonstrated the simulation with 6 packet/minute and it will exhaust the battery faster than 1 packet/ minute.

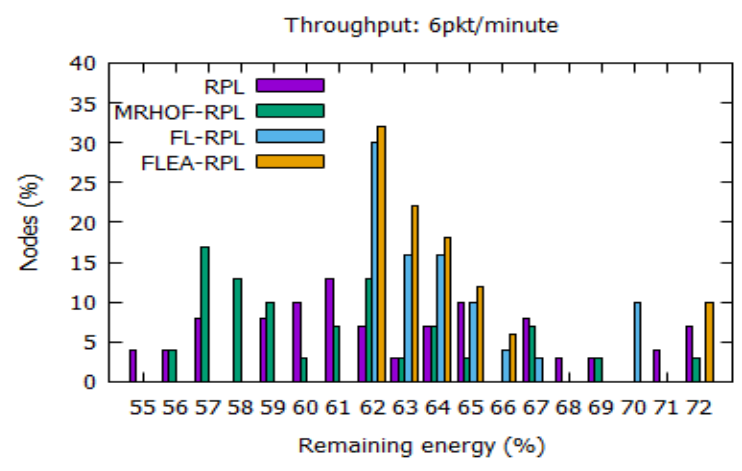

Fig.9. Remaining power distribution for 6 packet/minute

\section{C4. Average Packet Loss Ratio}

We conducted the simulation and observed that FLEARPL protocol provides lower value of average packet loss than other similar RPL protocol with throughput 1 to 6 packet per minute. RPL based network lead to high packet loss, due to it does not promote the link quality. Hence, the parent node may be congested or drop the packet during the data transmission from source to destination. ETX based network follows the high link quality for choosing the parent node but the packet loss happens, due to energy exhaustion. FL-RPL based network comparatively reduced the average packet loss ratio than RPL, MRHOF-RPL. In FL-RPL, When the network size is increasing, the packet loss ratio also increased automatically. Fig.10 shows that an average packet loss ratio with respect to network size. FLEA-RPL with throughput 1 packet per minute and the simulation result shows that there is no packet loss ratio up to the network size is 40 . Packet loss ratios are $1 \%, 3 \%, 4 \%$ for the network size 60, 80 and 100 respectively.

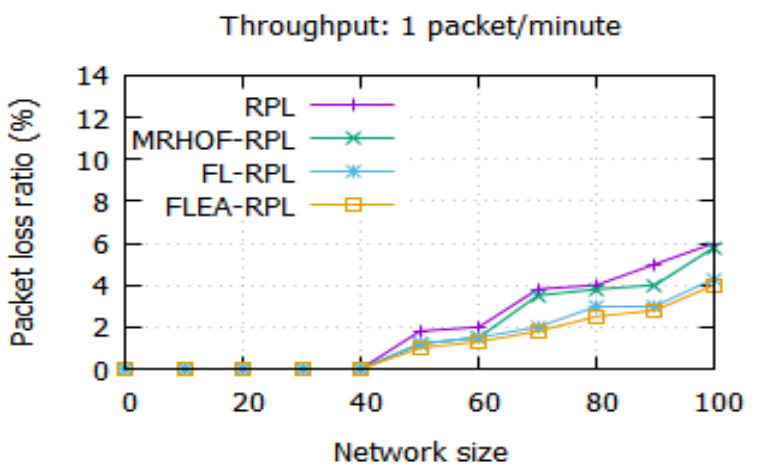

Fig.10. Average packet loss ratio vs. different network size 
Fig.11 shows that an average packet loss ratio with respect to network size. FLEA-RPL with throughput 6 packet per minute simulation result shows that there is no packet loss ratio up to the network size is 20. Packet loss ratios are $1 \%, 2 \%, 4 \%$ and $7 \%$ for the network size 40,60 , 80 and 100 respectively.

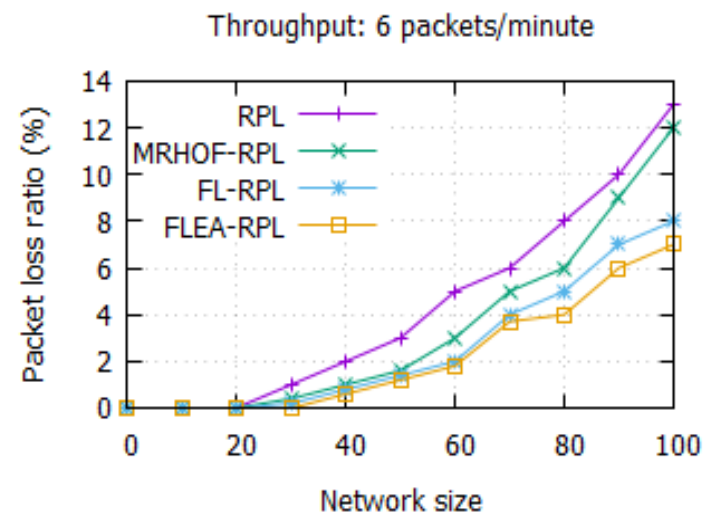

Fig.11. Average packet loss ratio vs. different network size

\section{C5. Effect of the Network Node Failure on Packet Loss Ratio}

We conducted the simulation of the proposed FLEARPL in the presence of node failure. We varied the throughput values from 1 packet / minute to 6 packet / minute. Fig. 12 shows that an average number of packet loss ratio of different number of failure happened node. We observed the simulation results, the number of failed node increases at the same time the number packet loss ratio is also increased. The FLEA-RPL provided the lower packet loss ratio than other similar routing protocol RPL, MRHOF-RPL and FL-RPL. RPL based network does not consider the link quality. MRHOF-RPL based network increases the node failure, due to the early battery exhaustion. FL-RPL based network does not consider the traffic load. The number of failed node size is 30 and average packet loss ratios are 15, 10, 7, 6 for RPL, MRHOF-RPL, FL-RPL and FLEA-RPL respectively.

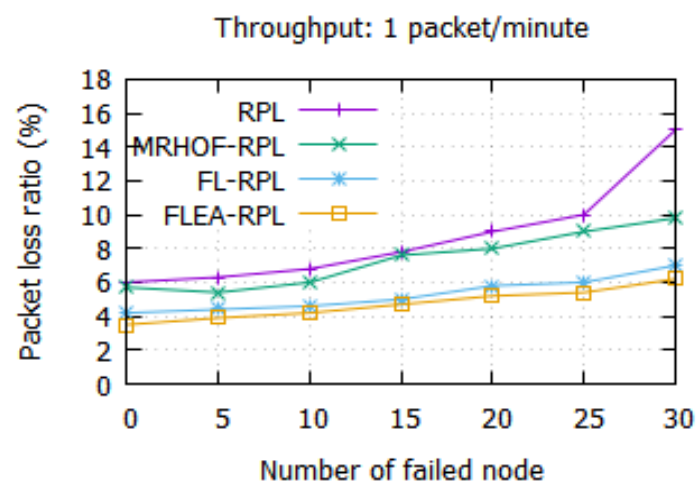

Fig.12. Average packet loss ratio vs. Number of failed node

Fig.13 shows that packet loss ratio with 6 packet/ minute with respect to number of failed node in the network. The number of failed node is 30 , an average packet loss ratios are 21, 18, 15, and 13.5 for RPL, MRHOF-RPL, FL-RPL and FLEA-RPL respectively.

\section{Throughput: 6 packets/minute}

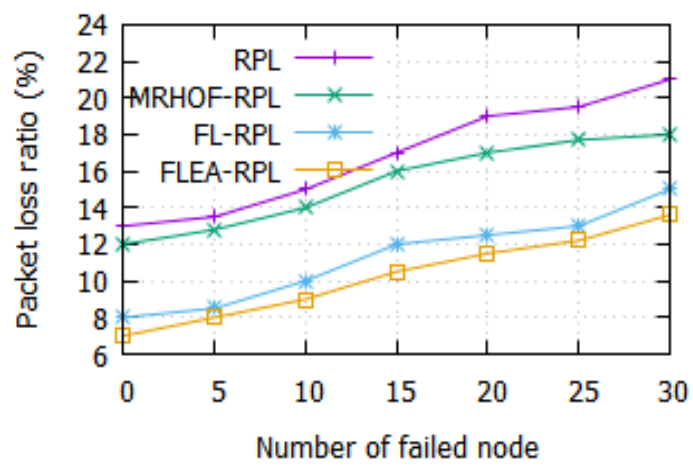

Fig.13. Average packet loss ratio vs. Number of failed node

\section{CONCLUSION}

In this paper, we proposed a fuzzy logic based energy aware RPL protocol for Internet of Things. In FLEA-RPL participant node wants to send the data to DODAG root through parent node. It chooses the best parent based on the quality, among its preferred parents in DODAG. The quality of parent node is calculated by applying the fuzzy logic over the routing metrics load, ETX and RER of the node. The node with the high quality value among the preferred parents is selected as the parent node to transfer the data, in DODAG. The simulation result shows that FLEA-RPL provides the better performance in terms of the packet delivery ratio and network lifetime compared to RPL, MRHOF-RPL and FL-RPL.

As part of future work, it is planned to provide mobility to the nodes, in low power and lossy networks (LLN) and it is also planned is to deploy it in the real time environment.

\section{REFERENCES}

[1] Lin, Jie, Wei Yu, Nan Zhang, Xinyu Yang, Hanlin Zhang, and Wei Zhao. "A survey on internet of things: Architecture, enabling technologies, security and privacy, and applications." IEEE Internet of Things Journal, Vol.4, Issue.5, pp: 1125-1142, 2017.

[2] Gazis, Vangelis. "A Survey of Standards for Machine-toMachine and the Internet of Things." IEEE Communications Surveys \& Tutorials, Vol.19, Issue.1, pp: 482-511, 2017.

[3] S. Sankar and P. Srinivasan, "Internet Of Things (Iot): A Survey On Empowering Technologies, Research Opportunities And Applications,'International Journal of Pharmacy and Technology, Vol. 8, pp.26117-26141, Dec 2016.

[4] S.Sankar and P.Srinivasan,"Composite Metric Based Energy Efficient Routing Protocol for Internet of Things,"International Journal of Intelligent Engineering and Systems, Vol.10, Issue.5, pp. 278-286, 2017.

[5] Kim, Hyung-Sin, Jeonggil Ko, David E. Culler, and Jeongyeup Paek. "Challenging the IPv6 Routing Protocol for Low-Power and Lossy Networks (RPL): A Survey." 
IEEE Communications Surveys \& Tutorials, Volume.19, Issue.4, pp: 1-24, 2017.

[6] Mohamed, Belghachi, and Feham Mohamed, "QoS Routing RPL for Low Power and Lossy Networks." International Journal of Distributed Sensor Networks, Vol.11, Issue.11, pp.971545, 2015.

[7] Thang, Vu Chien, and Nguyen Van Tao, "A Performance Evaluation of Improved IPv6 Routing Protocol for Wireless Sensor Networks," International Journal of Intelligent Systems and Applications, Vol.8, Issue.12, pp.18, 2016.

[8] T. Winter, "RPL: IPv6 Routing Protocol for Low-Power and Lossy Networks",https://tools.ietf.org/html/rfc6550, 2012.

[9] Iova, Oana, Pietro Picco, Timofei Istomin, and Csaba Kiraly, "RPL: The Routing Standard for the Internet of Things... Or Is It?," IEEE Communications Magazine, Vol.54, Issue. 12 pp. 16-22, 2016.

[10] Qasem, Mamoun, Ahmed Al-Dubai, Imed Romdhani, Baraq Ghaleb, and Wajeb Gharibi, "A New Efficient Objective Function for Routing in Internet of Things Paradigm," In Standards for Communications and Networking (CSCN), 2016 IEEE Conference on, pp. 1-6., 2016.

[11] Wang, Zhenfei, Liying Zhang, Zhiyun Zheng, and Junfeng Wang. "Energy balancing RPL protocol with multipath for Wireless sensor networks." Peer-to-Peer Networking and Applications, pp: 1-16, 2017.

[12] Hassan, Ali, Saleh Alshomrani, Abdulrahman Altalhi, and Syed Ahsan, "Improved Routing Metrics for Energy Constrained Interconnected Devices in Low-Power and Lossy Networks," Journal of Communications and Networks, Vol.18, Issue. 3, pp.327-332,2016.

[13] Nassiri, Mohammad, Mohammad Boujari, and Seyed Vahid Azhari,"Energy-Aware and Load-Balanced Parent Selection in RPL Routing for Wireless Sensor Networks." International Journal of Wireless and Mobile Computing, Vol. 9, Issue. 3 pp.231-239, 2015.

[14] Tian, Hongliang, Zhihong Qian, Xue Wang, and Xiao Liang. "QoI-Aware DODAG Construction in RPL-Based EventDetection Wireless Sensor Networks." Journal of Sensors, pp: 1-9, 2017.

[15] Shakya, Nikesh Man, Mehdi Mani, and Noel Crespi. "SEEOF: Smart energy efficient objective function: Adapting RPL objective function to enable an IPv6 meshed topology solution for battery operated smart meters." In Global Internet of Things Summit (GIoTS), IEEE, pp. 1-6, 2017.

[16] Gaddour, Olfa, Anis Koubâa, and Mohamed Abid, "Quality-of-Service Aware Routing for Static and Mobile IPv6-Based Low-Power and Lossy Sensor Networks using RPL," Ad Hoc Networks, Vol.33, pp.233-256,2015.

[17] Alvi, Sheeraz A., Fakhar ul Hassan, and Adnan Noor Mian. "On the energy efficiency and stability of RPL routing protocol." In Wireless Communications and Mobile Computing Conference (IWCMC), 2017 13th International, IEEE, pp. 1927-1932, 2017.

[18] De Couto, Douglas SJ, Daniel Aguayo, John Bicket, and Robert Morris, "A high-Throughput Path Metric for Multi-Hop Wireless Routing," Wireless networks, Vol.11, Issue. 4, pp: 419-434, 2005.

[19] Balaji, S., E. Golden Julie, and Y. Harold Robinson. "Development of Fuzzy based Energy Efficient Cluster Routing Protocol to Increase the Lifetime of Wireless Sensor Networks." Mobile Networks and Applications, pp: 1-13, 2017.

[20] Mittal, Poonam, Mehak Jain, C. K. Nagpal, and
Shailender Gupta, "A Throughput and Spectrum Aware Fuzzy Logic Based Routing Protocol for CRN." International Journal of Computer Network and Information Security, Vol.8, Issue.3, pp. 58, 2016.

[21] Mohamed Ababou, Mostafa Bellafkih and Rachid El kouch." Energy Efficient Routing Protocol for Delay Tolerant Network Based on Fuzzy Logic and Ant Colony", International Journal of Intelligent Systems and Applications, Vol. 10, issue. 1,pp: 69-77, 2018.

[22] Zhao, Liang, Ahmed Y. Al-Dubai, and Geyong Min, "An Efficient Neighbourhood Load Routing Metric for Wireless Mesh Networks." Simulation Modelling Practice and Theory, Vol.19, Issue. 6, pp.1415-1426, 2011.

[23] Izquierdo, Segismundo S., and Luis R. Izquierdo. "Mamdani Fuzzy Systems for Modelling and Simulation: A Critical Assessment." 2017.

[24] Alhumaidi, Hanouf M. "Fuzzy weighted average approach to ranking projects in contractor initial bidding." Fuzzy Information Processing Society (NAFIPS), 2016 Annual Conference of the North American. IEEE, pp: 1-7, 2016.

\section{Authors' Profiles}

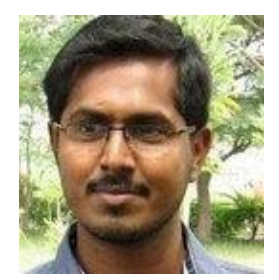

S.Sankar completed Master of Engineering in Computer Science and Engineering from Anna University, Tamilnadu, India. Currently I am pursuing full time $\mathrm{PhD}$ in Internet of Things at Vellore Insitute of Technology, Vellore. India. Research interest includes IoT Routing Protocol and IoT Analytics.

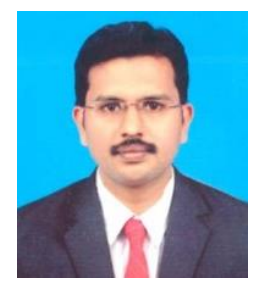

Srinivasan $\mathbf{P}$ received M.C.A degree from Periyar University and Ph.D. from Anna University, India in 2014. He is currently working as an Associate Professor in Vellore Insitute of Technology, Vellore. Research interest includes Cloud Computing, Wireless Networks, Smart and Pervasive Computing, IoT and Machine Learning. Published various papers in International journals and conferences. Has 13 years of teaching experience in reputed engineering colleges in Tamil Nadu and Karnataka. Coordinated and attended various Faculty Developments Programmes and International conferences.

How to cite this paper: S.Sankar, P.Srinivasan, "Fuzzy Logic Based Energy Aware Routing Protocol for Internet of Things", International Journal of Intelligent Systems and Applications(IJISA), Vol.10, No.10, pp.11-19, 2018. DOI: 10.5815/ijisa.2018.10.02 